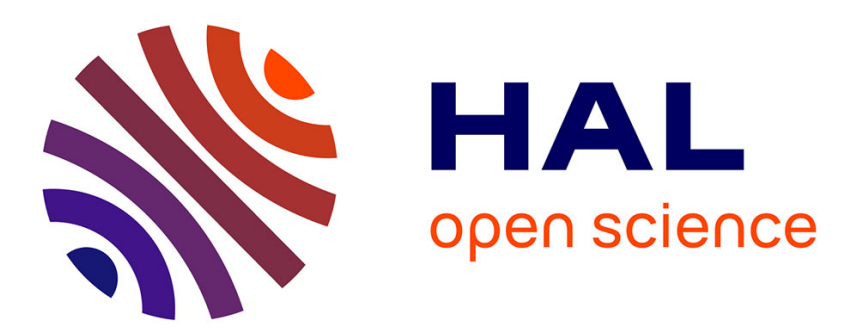

\title{
Mondialisation et développement. Quelle soutenabilité au régime de l'organisation mondiale du commerce?
}

\author{
Mehdi Abbas
}

\section{To cite this version:}

Mehdi Abbas. Mondialisation et développement. Quelle soutenabilité au régime de l'organisation mondiale du commerce?. Mondes en Développement, 2011, 154, pp.17-28. 10.3917/med.154.0017 . halshs-00602996

\section{HAL Id: halshs-00602996 \\ https://shs.hal.science/halshs-00602996}

Submitted on 28 Jun 2011

HAL is a multi-disciplinary open access archive for the deposit and dissemination of scientific research documents, whether they are published or not. The documents may come from teaching and research institutions in France or abroad, or from public or private research centers.
L'archive ouverte pluridisciplinaire HAL, est destinée au dépôt et à la diffusion de documents scientifiques de niveau recherche, publiés ou non, émanant des établissements d'enseignement et de recherche français ou étrangers, des laboratoires publics ou privés. 


\title{
ÉCONOMIE DU DÉVELOPPEMENT DURABLE ET DE L'ÉNERGIE
}

\section{Mondialisation et développement}

\author{
Quelle soutenabilité au régime de \\ l'Organisation mondiale du commerce?
}

Mehdi Abbas

avril 2011

Cahier de recherche $n^{\circ} 4 / 2011$ 



\section{Mondialisation et développement.}

\section{Quelle soutenabilité au régime de l'Organisation mondiale du commerce?}

Mehdi Abbas, maitre de conférences, Université de Grenoble, LEPII, CNRS.

Université Pierre Mendès-France

38040 Grenoble Cedex 9

Tel. : 0456528593

Courriel : mehdi.Abbas@upmf-grenoble.fr

\section{Résumé}

Cet article présente les principales contradictions dans lesquels est prise l'Organisation mondiale du commerce tel que les révèle le déroulement du programme de Doha pour le développement. Ils constituent une manifestation des contradictions structurelles et institutionnelles dans les rapports NordSud au sein du système commercial multilatéral. L'article réfute la thèse du nombre comme explication prédominante de l'enlisement de la négociation. L'articulation entre la nature de la régulation commerciale contenue dans les Accords de l'OMC et l'évolution des équilibres de puissance constitue la source majeure des dysfonctionnements du système commercial multilatéral. Il en résulte un abandon de la dimension développement du cycle de Doha et une interrogation sur la soutenabilité environnementale et sociale du régime commercial de l'OMC.

Mots-clés : multilatéralisme, libre-échange, émergence, traitement spécial et différencié. 


\section{INTRODUCTION}

La quatrième Conférence Ministérielle de l'OMC, tenue du 9 au 14 novembre 2001, a lancé le programme de Doha pour le développement (PDD). Celui-ci est une négociation, placée sous le sceau de l'engagement unique, dont le principal objectif est l'intégration des PED-PMA au «système commercial multilatéral ouvert [et] fondé sur des règles [dans des conditions qui] correspondent aux besoins de leur développement économique »(OMC, 2001).

En lançant un cycle pour le développement les Etats Membres ont décidé de traiter les déséquilibres initiaux résultant des Accords du cycle d'Uruguay dont souffraient les économies du Sud et signifié que la libéralisation n'est pas la finalité ultime des négociations. Mais, force est de constater que ce programme ambitieux (21 thèmes de négociations) peine à aboutir. Cet «agenda en contraction » (Winham, 2007) s'est progressivement transformé en une négociation commerciale classique ayant pour principale thématique l'accès au marché et le démantèlement de barrières non tarifaires (soutien interne et subvention).

L'objet de cette contribution est de fournir une analyse de l'enlisement du PDD qui dépasse le réductionnisme de la thèse du nombre conduisant à considérer que les problèmes que rencontre le système commercial multilatéral relèvent de la procédure de votation et de la règle du consensus ${ }^{1}$. Cette lecture permet d'imposer l'idée selon laquelle si le multilatéralisme ne fonctionne pas, c'est en raison des "won't do countries » ou des "paralyzzers countries »². Or, le problème n'est pas tant "la capacité de l'OMC à mener son mandat » (Blackhurst, 1998) que le contenu et les implications de ce mandat, c'es-à-dire la substance du régime commercial multilatéral de l'OMC.

L'enlisement du cycle de Doha et les défaillances du système commercial multilatéral sont la manifestation des contradictions systémiques dont est porteur le régime de commerce, d'investissement et de développement de l'OMC. L'importance prise par les crises écologiques et alimentaires à quoi s'ajoutent les déséquilibres macroéconomiques globaux révélés par la crise financière de 2007 convergent pour poser un problème de soutenabilité du régime issue du cycle d'Uruguay (1986-1994). Le contenu de ce problème se décline au travers deux dysfonctionnements. D'une part, l'articulation défaillante entre la substance et le mode opératoire du régime OMC donne lieu aux dysfonctionnements du multilatéralisme compétitif. D'autre part, une contradiction entre la substance du sous-régime de commerce et développement et l'évolution de l'équilibre de puissance qui empêche l'élaboration d'une doctrine satisfaisante alliant mondialisation et développement.

Cet article s'organise en trois parties. La première porte sur la dimension institutionnelle de l'enlisement du PDD, c'est-à-dire le contenu du régime de l'OMC. La deuxième prolonge cet aspect en analysant les contradictions de l'articulation entre mondialisation et développement contenu dans le régime OMC. La troisième partie montre en quoi les contradictions précédemment exposées amplifient la difficulté historique du régime OMC à adresser correctement la question du développement durable.

\section{L'INCAPACITE A RENDRE OPERATIONNEL LE REGIME DE L'OMC}

$\mathrm{Si}$, dans un premier temps, les analyses ont insisté sur les éléments de continuité entre le GATT et l'OMC, il apparaît désormais que cette dernière marque un changement de nature dans la régulation multilatérale des échanges. Cette appréciation découle de deux séries de facteurs.

$\mathrm{La}$ première renvoie au fait qu'avec l'OMC le système commercial multilatéral aborde la troisième génération d'obstacles au échanges ${ }^{3}$. Les Accords de l'OMC ne portent plus uniquement sur les protections aux frontières mais concernent de façon croissante les « mesures au-delà des frontières » et les dispositifs réglementaires et institutionnels ${ }^{4}$. Les questions de subventions, de propriété intellectuelle, de

\footnotetext{
${ }^{1}$ Voir Porter, 2001 ; WTO, 2004 ; Cass, 2005 ; Petersmann, 2005 ; Warwick Commission, 2007 ; Steger, 2010.

${ }^{2}$ Expressions utilisées par les négociateurs de la délégation américaine lors de la conférence ministérielle de Cancun. Voir Bello, 2003 et Taylor, 2007.

${ }^{3}$ Les première et deuxième générations concernaient les droits de douane et les barrières non tarifaires. La troisième génération concerne les systèmes nationaux et souverains de régulation.

${ }^{4}$ A savoir : les mesures techniques, les règles et procédures administratives, normatives et d'expertise, les restrictions quantitatives et réglementaires à l'importation, les taxes internes, les restrictions en matière de concurrence et de liberté de circulation, et les exigences en matière d'étiquetage.
} 
services et de marchés publics relèvent des déterminants de la compétitivité structurelle des économies. Dès lors, l'une des fonctions de la négociation OMC est de créer les conditions de la contestabilité en réduisant les contraintes juridiques, institutionnelles (brevets, normes sanitaires, techniques, labels) et stratégiques mises en place par les Etats, les politiques de libéralisation et d'ouverture économiques étant associées à une réduction des barrières à l'entrée d'un marché (Graham, Lawrence, 1996). Le régime OMC inaugure une nouvelle place pour les normes dans la prévention des entraves techniques au libre-échange faisant que la contestabilité des marchés porte sur les politiques non commerciales et les différences dans les régimes de régulation (politiques environnementale et de concurrence, standards de protection de la propriété intellectuelle) et les barrières qualitatives aux échanges.

La seconde série de facteurs, de nature réglementaire, découle de l'observation faite par S. Ostry (2007), qui relève que les Accords du Cycle d'Uruguay, l'AGCS et l'ADPIC en particulier, substituent à une régulation négative - c'est-à-dire ce qu'un gouvernement ne peut pas faire - une régulation positive c'est-à-dire ce qu'il doit faire. L'agenda de l'OMC comporte un nouveau corpus de règles avec des recommandations de mise en conformité, des normes de procédures, des normes substantives ${ }^{5}$.

Ces deux séries de facteurs font que le système commercial multilatéral est passé d'une logique où il s'agissait de réguler les tendances protectionnistes des Etats Membres à une logique d'incitation à la libéralisation. Celle-ci se trouve consolidée par la multiplication des questions relevant du " "Trade and..." agenda 》 (commerce et investissement, commerce et concurrence, commerce et environnement, commerce et standard technique) (Hoeckman, Kostecki, 2009).

Mais ce que nous révèle l'évolution du PDD c'est que le multilatéralisme de l'OMC peine à trouver son mode opératoire pour au moins trois raisons.

Premièrement, les Etats membres n'ont, semble-t-il, pas pris la mesure de la difficulté et de la complexité technique de négociations où les questions commerciales sont périphériques (Baldwin, 2006). Les quinze ans de l'OMC constitueraient une période d'apprentissage de négociations portant sur les normes, la diversité institutionnelle et les préférences collectives. Cette évolution conduit à une confrontation entre plusieurs corps de normes juridiques et à une réflexion sur la connexion et la hiérarchie à établir entre les différents systèmes normatifs (juridique, politique, technologique, culturel, social, commercial). C'est un chantier d'une redoutable complexité par rapport auquel il n'existe pas de consensus entre les membres.

Deuxièmement, la nature multisectorielle des négociations, qui dans le cadre de négociations classiques garantit l'obtention d'un compromis entre les offres et demandes d'accès aux marchés, est ici inopérante. La complexité des problèmes concurrentiels, normatifs et réglementaires implique que les négociations soient menées sur une base sectorielle. L'espace disponible pour un compromis s'en trouve réduit. Force est de constater que les schémas d'analyse de Krugman (1997), Bagwell et Staiger (1999) et Hoeckman et Kostescki (op. cit.) mettent en avant la rationalité procédurale et non substantive des négociations. Ils ne permettent pas de rendre opérationnelle une négociation ne portant pas essentiellement sur l'échange réciproque de concessions tarifaires. On ne dispose toujours pas de cadre analytique en mesure de le rendre effectif. C'est bien cet aspect qui explique le retrait des dossiers concurrence et investissement, d'autant plus que les gains attendus par cette négociation ne sont pas démontrés (Panagariya, 2002).

Troisièmement, la contestabilité internationale des marchés fait que le régime de l'OMC a compétence sur l'action régulatrice des Etats, ce qui a pour effet de circonscrire leur pouvoir discrétionnaire dans l'organisation de leur économie et d'encadrer des politiques qui relevaient auparavant de leur souveraineté dans la mesure où de telles politiques affectent leurs importations ${ }^{6}$. Par ailleurs, la procédure de règlement des différends expose de façon permanente les pays au risque d'une procédure. Le plus sûr moyen d'éviter ces deux phénomènes est de ne pas inscrire de nouveaux secteurs à l'ordre du jour de la libéralisation ou d'adopter une stratégie de blocage. Elle confère aux arbitres du commerce multilatéral un pouvoir de sélection et de validation des normes, qui échappe à la diplomatie commerciale des Etats et réduit considérablement leur autonomie réglementaire (Balmelli, Chaisse, 2008).

A ces éléments endogènes à l'architecture commerciale multilatérale s'ajoutent les critiques adressées au paradigme du libre-échange, matrice théorique de la mondialisation. Le PDD est lancé au moment où ce

\footnotetext{
${ }^{5}$ Il s'agit des articles 2.2 et 2.4 de l'accord sur les obstacles techniques au commerce, 3.3 et 5 de l'accord sur les mesures sanitaires et phytosanitaires, les articles VIII, X du GATT de 1994. A cela s'ajoute que l'Omc régule le recours à des exemptions pour motifs non commerciaux (articles XX et XXI du GATT et XIV de l'AGCS.

6 Particulièrement de mesures sanitaires et phytosanitaires, environnementales, d'investissement, de recherche et développement, de régulation des monopoles nationaux, d'usages des terres, d’organisation du secteur énergétique.
} 
paradigme est contesté du fait de la crise asiatique et de la montée des inégalités au sein des pays développés principalement. La croyance en un commerce international systématiquement bénéficiaire n'est plus théoriquement fondée dès lors que les marchés sont imparfaits et que les acteurs ont des stratégies non réductibles aux principes de l'avantage comparatif. A côté du conflit distributif associé à la montée des inégalités, la mondialisation donne lieu à une redistribution des spécialisations, dont l'émergence est l'aspect le plus visible, source à son tour de contestation de la libéralisation.

Jusqu’à la conférence de Cancun, les négociations fonctionnaient selon une logique oligopolistique (la Quadrilatérale), voire duopolistique (Etats-Unis et Union européenne). Or, ce modèle avec inclusion par palier des économies de second rang en vue d'obtenir une masse critique avant que le compromis ne soit proposé à l'ensemble des pays membres, qui a fait ses preuves durant près d'un demi-siècle, est pris en défaut. Graduellement, le modèle de club (peu de membres décidant pour tous les membres) cède la place à un modèle de coalitions dans lequel un ensemble de membres décidant pour tous les membres. Ces réseaux de coalitions se forment sur une base d'intérêts sectoriels autours de question d'accès au marché et non plus sur une base idéologique ou géopolitique.

En effet, l'un des enseignements du PDD est qu'il n'existe pas nécessairement de synergie et encore moins une communauté d'intérêt entre ce G3 élargi à un G20 et le G99. Ainsi, la Conférence Ministérielle de Genève a-t-elle vu s'opposer le groupe Afrique à la FIP (Etats-Unis, Union européenne, Brésil, Inde, Australie) et le G33 au G20 (Iqbal, 2008) ${ }^{7}$. Aussi, la séquence allant de Cancun à Hong Kong impose de distinguer le «Sud Global» (Brésil, Chine et Inde), qui mérite une attention particulière compte tenu de son potentiel concurrentiel et de sa capacité compétitive sur l'ensemble de la chaîne de valeur et non uniquement sur les activités intensives en main-d'œuvre ou en ressources (Bello, 2005 ; Yusuf et al., 2007).

De plus, les externalités environnementales et climatiques accompagnent la prise de conscience d'un écart sans cesse croissant entre les gains privés du libre-échange et les coûts collectifs que la libéralisation peut générer (dégradation de l'environnement, croissance des émissions de $\mathrm{CO}_{2}$, déséquilibres régionaux, surexploitation agricole, destruction d'emplois, etc.). Et de fait l'OMC n'est plus en mesure de démontrer que la libéralisation multilatérale des échanges constitue une réponse soutenable et collective aux défis de l'intégration économique internationale (problèmes environnementaux, inégalités, délocalisations, instabilité monétaire et financière).

Le PDD aurait dû et pu être l'occasion d'une réflexion approfondie sur les effets adverses de la mondialisation. Or, il reste fondé sur l'hypothèse que les Etats Membres s'engagent dans les négociations uniquement en vue d'élargir et d'approfondir la libéralisation commerciale multilatérale (Jones, 2010). Cette confusion permanente entre multilatéralisme et libre-échange, qui date du cycle d'Uruguay, s'avère préjudiciable au système

Le régime de l'OMC se trouve ainsi pris en défaut vis-à-vis des évolutions structurelles du système commercial international. Celui-ci est désormais déterminé par des tendances à l'intégration et par les mises en réseaux des structures productives. Cette contradiction entre une économie mondiale intégrée de façon croissante par les réseaux de firmes et des négociations de nature strictement interétatique expliquerait les défaillances du multilatéralisme en tant que régime de construction d'un système de droit international sur un mode intergouvernemental (Deblock, 2010). Cette contradiction est amplifiée par le fait que l'OMC cloisonne les thématiques alors que le système commercial multilatéral est désormais un système intégré au sens où il y a convergence entre politique commerciale et politique sociale et environnementale, entre politique commerciale et politique technologique et bien entendu entre politique commerciale et politique de développement.

Cela nous conduit à traiter de la deuxième contradiction que révèle le déroulement du PDD, celle de l'impossibilité d'élaborer un paradigme opérationnel de la relation mondialisation-développement.

\section{LES LIMITES DE L'ARTICULATION MONDIALISATION-DEVELOPPEMENT DU REGIME DE L'OMC}

Le premier élément illustrant ce dilemme réside dans les résultats décevants en matière de gains commerciaux résultant des engagements souscrits jusqu'à présent dans le cadre du PDD.

\footnotetext{
${ }^{7}$ Avant la Ministérielle de Hong Kong, l’Inde, le Brésil et l’Afrique du Sud ont soumis une proposition « commerce et développement » concernant l'agriculture, l'AMNA, l'accord anti-dumping, les mesures d'investissement liées au commerce et l'ADPIC comportant des dispositions favorables aux PED. La Chine ne s'est pas associée à cette initiative, qui reste la dernière en date en provenance de PED et à l'adresse d'autres PED.
} 
Partant d'une projection initiale estimant que la négociation améliorerait le revenu mondial de 520 milliards de \$, la Banque mondiale a révisé ses estimations pour établir que le gain serait de 287 milliards dont «seulement» 90 milliards pour les pays en développement (Hertel, Winters, 2005). En 2005, date censée conclure le Programme, la Banque mondiale produisait une nouvelle estimation des «bénéfices » de la négociation chiffrée à 100 milliards de $\$$, dont 18 milliards seulement pour les PED (Anderson et al., 2005) ${ }^{8}$. Les estimations de l'Institut Peterson à l'occasion de la Conférence Ministérielle de Genève (2009), indiquent que le PIB mondial augmentera de $0,2 \%$ en se répartissant à hauteur de $66 \%$ pour les pays développés et $34 \%$ pour les PED (Alder et al., 2009). D'autres travaux indiquent que les gains commerciaux attendus seraient de 99 milliards de $\$$ tout en admettant que leurs calculs ne prennent pas en compte les coûts de la libéralisation et que des investissements significatifs seraient nécessaires dans les PED pour que ces gains se réalisent (Minor, Tsigas, 2008). Quant à Decreux et Fontagné (2009), ils pronostiquent un gain de 57 milliards de $\$$ pour l'agriculture et l'accès au marché industriel, et de 11 milliards de \$ pour les services. Ils soulignent que l'aide au développement décidée à Hong Kong permettrait un gain potentiel de 100 milliards de $\$$, ce qui rejoint la projection de la Banque mondiale. Mais ce résultat est également conditionné par d'importants investissements que le PDD ne prévoit pas.

Cette faiblesse des gains attendus est amplifiée par leur inégale répartition. Les travaux convergent tous pour indiquer que les pays qui bénéficieraient le plus d'un accord en l'état des propositions mises sur la table des négociations sont l'Argentine, le Brésil, la Chine, l'Inde, le Mexique, la Thailande, la Turquie et le Vietnam (Polski, 2006). Outre l'absence de critères et de finalité quant au contenu développement du Programme évoqué plus haut, un nouveau défaut surgit. Avoir lancé un programme pour le développement signifiait d'entrée de jeu qu'un ensemble de pays - les PED - allait "gagner » plus que d'autres; encore eût-il fallu préciser quels étaient les PED concernés et veiller à ce que les gains soient équitablement répartis entre eux ? En effet, la distribution inégale des gains attendus de la négociation ne crée aucune incitation à la ratification d'un compromis?.

De surcroît la libéralisation commerciale induirait une perte de revenu douanier - et donc une perte de capacité fiscale et redistributive - pour les pays en développement, estimée à 63,4 milliards de \$ par Cordoba et Vanzetti (2006). L'argument de la perte de revenu fiscal ne doit pas être sous-estimé dans des pays où les droits de douane constituent l'instrument de taxation le plus efficace et la principale source de financement des gouvernements (Emran, Stiglitz, 2005). Ce constat résulte de la transformation du PDD en une négociation commerciale où la dimension développement est ramenée soit à l'équilibre gain/coût de la libéralisation commerciale (Gallagher, 2007) soit aux effets concurrentiels positifs de la réduction tarifaire, voire à une articulation entre le Traitement Spécial et Différencié et l'Aide au Commerce (Ismail, 2007). Or cela n'est suffisant ni pour répondre aux enjeux du développement ni pour redresser l'inéquité du système.

Le "paquet développement» issu de la conférence ministérielle de Hong Kong (décembre 2005) - et qui reste à ce jour la dernière proposition sur la table de négociation - consiste à fournir un accès au marché des pays industrialisés aux pays les plus pauvres membres de l'OMC ( $97 \%$ des exportations de 90 pays membres). On en revient à une logique de préférences commerciales ciblées, sélectives et sans obligation juridique d'octroi concernant essentiellement les pays les moins avancés. Le paquet admet des actions spécifiques sur quelques produits de base et une aide au commerce dont la portée opérationnelle reste à déterminer. Cette victoire de l'interprétation commerciale de la déclaration de Doha est synonyme de l'échec dans la forme et la substance d'une négociation orientée vers le développement (Wilkinson, 2007).

L'OMC demeure dans la logique héritée de la partie IV du GATT comme si une force de rappel empêchait l'Institution d'envisager autre chose que l'octroi de préférences commerciales. Cette logique défendable dans un contexte protectionniste (1950-1985) ne l'est plus aujourd'hui. Pour leur part, les PED-PMA souhaitent que l'OMC serve de levier en matière d'aide au commerce et de mise en cohérence des différents mécanismes multilatéraux d'aide au commerce. Selon eux, le système commercial multilatéral doit servir à la construction de capacités commerciales, à équilibrer les règles de sorte qu'elles garantissent un accès équitable aux marchés pour leurs exportations (Ismail, 2005) ${ }^{10}$. Par delà le problème du nombre,

\footnotetext{
${ }^{8}$ Pour une analyse critique des méthodes et des conclusions analytiques de ces estimations voir Ackerman, 2005.

${ }^{9}$ Le gain de la libéralisation agricole bénéficierait pour $90 \%$ au Brésil, Mexique, Thaïlande et Philippines (Anderson, Martin, 2006).

${ }^{10}$ On notera l'introduction du terme d'équité des règles et de l'accès au marché concernant les PED-PMA sans précision ni sur le sens ni sur le contenu du terme qui semble se substituer à l'idée d'un traitement spécial et différencié.
} 
l'enlisement du PDD et l'impossibilité de trouver un compromis tient au fait que le Sud global n'est plus disposé à accepter les termes d'un compromis aussi asymétrique que ceux du Cycle d'Uruguay.

Le dossier agricole illustre les dilemmes de cette situation. Lancée en 2001, la négociation visait la résolution du problème des surproductions structurelles alors que désormais la question agricole mondiale se pose en termes d'augmentation de la production avec diversification de l'offre et des zones de production pour répondre aux enjeux de sécurités alimentaire et écologique (Mattoo, Subramanian, 2008 ; Pisani, Chatellier, 2010). Or l'agenda de la négociation reste très éloigné de ces préoccupations. Par ailleurs, pourquoi l'idée d'un espace politique pour le développement a-t-elle été balayée d'un revers de la main alors qu'elle offrait un complément à l'approche de l'OMC (Abbas, 2006) ?11 Pense-t-on réellement que la résolution de problèmes comme les changements climatiques, la sécurité alimentaire, la lutte contre la pauvreté passe uniquement par des politiques de libéralisation commerciale ?

Peut-on penser une stratégie de développement ou de croissance pro-pauvres en faisant abstraction de l'ouverture, de la globalisation, de l'organisation internationale des filières de production et d'échange et des nouveaux équilibres internationaux comme tentent de l'imposer certains membre, relayés par le Secrétariat? L'OMC est certes une organisation du commerce, mais dans une économie globalisée où la politique commerciale possède des effets et des dimensions non commerciales.

\section{SOUTENABILITE DU REGIME DE COMMERCE ET DE DEVELOPPEMENT DE L’OMC}

L'OMC ne traite pas explicitement du développement durable. Certes le préambule instituant l'Organisation stipule que la libéralisation multilatérale des échanges permet "l'utilisation optimale des ressources mondiales conformément à l'objectif de développement durable, en vue à la fois de protéger et préserver l'environnement et de renforcer les moyens d'y parvenir d'une manière qui soit compatible avec leurs besoins et soucis respectifs à différents niveaux de développement économique » (GATT, 1994). Mais, le préambule n'a aucune valeur juridique contraignante. De fait l'OMC ne parvient pas à penser la soutenabilité environnementale et sociale de son régime de commerce et de développement exposé dans les deux précédentes sections. Elle se contente de reprendre à son compte les thèses développées dans la déclaration de Rio (1994) et l'Agenda 21 intitulé Promouvoir le développement durable par le commerce (Damian, Graz, 2001). Cette thèse étend la doctrine du développement extraverti à la protection de l'environnement et à la promotion du développement durable par la libéralisation des échanges. Dès lors, Tout en affirmant la primauté de la libéralisation des échanges sur la protection de l'environnement (Nordström, Vaughan, 1999) l'OMC fait de la libéralisation commerciale un levier de la protection de l'environnement (OMC, 2004). Le Comité du commerce et de l'environnement de l' OMC a eu lecture unilatérale de la relation et ignore les débats autours des conditions nécessaires pour atteindre un développement durable (Cole, 2000 ; Tisdell, 2000).

La thèse du soutien mutuel s'appuie sur deux arguments théoriques. La courbe environnementale de Kuznets (CEK) constitue le premier. Nous ne nous attarderons pas sur la fragilité de la démonstration de la CEK (Lekalis, 2000). Le second renvoie aux trois effets du libre-échange en matière d'environnement (Grossman, Krueger, 1993 ; Copeland, Taylor, 2003). L'effet d'échelle et l'effet composition du commerce international ont un effet indéterminé, l'effet technique joue favorablement car l'ouverture des frontières favorise l'investissement porteur de transferts de technologies propres.

Contrairement à B. Hoeckman (2005) nous ne soutenons pas la thèse selon laquelle l'OMC a évolué pour devenir une "organisation du commerce et du développement durable ». Tout d'abord, ce régime s'appuie sur une vision orthodoxe et instrumentale du rapport entre l'économie et la biosphère, celle-ci étant au service de la première. Les actions autorisées pour protéger ou sauvegarder les ressources naturelles et animales au titre de l'article XX du GATT-1994 sont envisagées comme des dérogations à la norme multilatérales et au droit à l'exportation. Le régime OMC est hermétique aux arguments soumettant la libéralisation commerciale aux impératifs de la préservation de la biosphère et à l'impossibilité de concilier libre-échange et internationalisation multilatérale des coûts environnementaux (Daly, 1996). Ensuite, la soutenabilité dépend de l'intensité du progrès technologique. Or, depuis S. Jevons et le "paradoxe du charbon » (1866) nous savons que le progrès technique ne réduit pas l'intensité de la ponction sur l'écosystème. Enfin, ce

\footnotetext{
${ }^{11}$ L'OMC a publié nombre de rapports en commun avec d'autres organisations ou agences multilatérales, sans que cela ne soit suivi d'effet (Оіт/Омс sur le travail et la mondialisation, Омs/Омс sur les politiques de santé dans la mondialisation, PNUE/OMC sur les changements climatiques). Il est regrettable que dix ans après le lancement d'une négociation consacrée au développement aucun rapport commun avec la CNUCED, l'ONUDi et/ou le PNUD n'ait été produit pour expliquer ce que serait une stratégie de développement en économie globalisée.
} 
régime évacue les conséquences du libre-échange multilatéral et de l'extraversion en termes de déstructuration des sociétés des pays du Sud, de perte de biodiversité, d'appauvrissement des sols en raison de la spécialisation. L'écart entre les coûts collectifs de la dégradation de l'environnement et les gains privés du libre-échange et son corollaire la croissance est évacué de l'analyse.

La thèse du soutien mutuel est réaffirmée à l'occasion de la publication du rapport OMC-PNUE (2009) sur la dimension commerciale de la lutte contre les changements climatiques. La libéralisation des biens et services environnementaux (paragraphe 31 de la Déclaration de Doha) y est présentée comme la voie privilégiées pour mettre «la politique commerciale au service du climat». Simultanément la consolidation de l'Adpic est présentée comme le meilleur moyen d'obtenir les technologies en mesure d'engager les pays du Sud sur un sentier de croissance verte. Or, le régime de l'OMC est fondé sur le paradigme de la mobilité des biens, des capitaux et des facteurs productifs qui implique une croissance des émissions de $\mathrm{CO}_{2}$. D'ailleurs, les travaux montrent clairement la corrélation positive entre libre-échange et croissance des émissions.

Ainsi, le discours de l'OMC n'a pas évolué malgré le changement de nature et d'échelle que constitue la problématique des changements climatiques et les enjeux de soutenabilité écologique que soulèvent le régime globalisé de croissance actuel (Abbas, 2010). L'émergence, en réduisant l'espace des spécialisations profitables et en accroissant la demande mondiale pour les ressources naturelles et agricoles, peut conduire à une surexploitation de ces ressources comme seul moyen à disposition des PED non émergents et des PMA pour retirer un gain de la globalisation, du moins du modèle de globalisation contenu dans les Accords de l'OMC.

Le PDD est sensé adresser la question des conflits et synergies de normes entre les accords multilatéraux d'environnement (AME) et les Accords de l'OMC. Les principaux problèmes identifiés sont: la discrimination de l'accès au marché pour motifs environnementaux, l'usage de mesures de politique commerciale pour obtenir la mise en œuvre de politique environnementale, l'élaboration d'instruments de politique commerciale permettant d'améliorer les gains environnementaux de la libéralisation et l'amélioration des synergies entre l'Adpic et les AME concernant le transfert de technologies propres. Aucun dossier ne semble connaitre d'avancer notable.

La cohérence de la gouvernance globale n'est pas de la seule responsabilité de l'OMC, mais il ne faudrait pas que la question de la soutenabilité du développement ne soit envisagée qu'au travers du prisme commercial. Cela passerait, d'une part, par une analyse critique de la thèse du soutien mutuel et, d'autre part, par un élargissement de la participation à la réflexion d'autres organisations. Cela est d'autant plus nécessaire que dans une économie internationale intégrée la politique commerciale possède des dimensions non commerciales appelant une réflexion sur ses interfaces avec la politique environnementale et sociale. D'ailleurs, le chantier de l'inter-normativité et de la hiérarchie entre les différents champs de la gouvernance globale devrait être prioritaire afin de mieux cerner les défaillances à traiter et les modalités pour le faire.

\section{CONCLUSION}

Le programme de Doha pour le développement en tant que projet de rénovation des rapports Nord-Sud et de «corrections » des asymétries héritées du cycle d'Uruguay n'existe plus. Cancun et les Conférences Ministérielles qui ont suivi lui ont substitué une négociation commerciale classique. Certes le "paquet global de Doba » (Lamy) sera ratifié. Est-ce pour autant que les contradictions qu'aura révélées le PDD seront levées? Nous avons tenté de mettre en évidence l'écart existant entre l'agenda de la négociation, les principales problématiques des relations commerciales internationales et l'état des rapports Nord-Sud.

Les Etats Membres ne pourront faire ni l'économie d'un débat sur les limites et les frontières du régime de l'OMC ni s'interroger sur sa soutenabilité sociale et écologique. Ils ne pourront éviter un débat sur les modalités de la coopération commerciale multilatérale et l'élargissement de cette coopération à d'autres forums et organisations internationales qu'impose la nouvelle géographie de la richesse et de la puissance issue d'une économie internationale de plus en plus intégrées. 


\section{BIBLIOGRAPHIE}

ABBAS M. (2010) Economie politique globale des changements climatiques, Grenoble, PUG.

AbBas M. (2006) Les rapports Nord-Sud et Sud-Sud dans le Cycle du développement de l'OmC, in H. Regnault, C. DeBlock (eds.) Intégration Nord-Sud et stratégies de développement, Montréal, Athéna.

ACKeRman F. (2005) The Shrinking Gains from Trade: a Critical Assessment of Doha round Projection, Working Paper, $\mathrm{n}^{\circ}$ 05-01, October, Medford, Global Development and Environment Institute, Tufts University.

Alder M. et al. (2009) What's on the Table? The Doha round as of August 2009, Working Paper, $\mathrm{n}^{\circ}$ 09-6, August, Washington, Peterson Institute for International Economics.

Anderson K., et al. (2005) Global Impacts of the Doha Scenario Poverty , in T. W. Hertel, A. Winters (eds.) Putting Development Back into the Doha Agenda: Poverty Impacts of a WTO Agreement, Washington, World Bank.

Anderson K., MARTin W.,eds. (2006) Agriculture Trade Reform and the Doha Development Agenda, Washington, World Bank.

Bagwell K., Staiger R. W. (1999) An Economic Theory of the GatT, American Economic Review, vol. 89, nº 1, 215 248.

BALDWIN R. (2006) Failure of the WTO Ministerial Conference at Cancun: Reasons and Remedies, The World Economy, vol. 29, n 6, 677-696.

Balmelli T., Chaisse J. (2008) The Future of the World Trade Organization and the Changing Structure of the International Legal System, in T. Balmelli, J. Chaisse (eds.) Essays on the Future of the World Trade Organization, Geneva, Edis.

BELlo W. (2005) The Real Meaning of Hong Kong: Brazil, And India Join Big Boys' Club, Focus on the Global South, 22 December.

BELLO W. (2003) Washington's Vendetta, [en ligne], disponible sur : http://www.geocities.com/ericsquire/articles/ftaa/frontline031025.htm [consulté le 24/04/2010].

Blackhurst R. (1998) The Capacity of the WTO to Fulfil its Mandate, in A. Krueger (ed.) The WTO as an International Organization, Chicago, Chicago University Press.

CAss D. (2005) The Constitutionalization of the World Trade Organization: Legitimacy, Democracy and Community in the International Trading system, Oxford, Oxford University Press.

Cole M. A. (1999) Limits to Growth, Sustainable Development and Environmental Kuznet's Curves: An Examination of the Environmental Impact of Economic Development, Sustainable Development, vol. 7, $\mathrm{n}^{\circ}$ 2, 8797.

Copeland B. R., Taylor M. S. (2003) Trade and the Environment, Princeton, Princeton University Press.

Cordoba S., VAnZETTI D. (2006) Now What? Searching for a Solution in WTO Industrial Tariff Negotiations, in S. Laird, S. Cordoba (eds.) Coping with Trade Reforms. A Developing Country Perspective on the WTO Industrial Tariff Negotiations, Basingstoke, Palgrave McMillan.

DALY H. (1996) Beyond Growth: the Economics of Sustainable Development, Boston, Beacon Press.

Damian M., GraZ J.C. (2001) Commerce international et développement soutenable, Paris, Economica.

DEBLOCK C. (2010) OMC : le déclin irréversible de la réciprocité et du multilatéralisme, L'économie politique, n 45, 3554.

Decreux Y., Fontagné L. (2009) Economic Impact of Potential Outcome of the DDA, Rapport d'études n 2009-1, Paris, CEPII.

Emran M. S., Stiglitz J. (2005) On Selective Indirect Tax Reform in Developing countries, Journal of Public Economis, vol. 89, $\mathrm{n}^{\circ}$ 4, 599-623.

GaLlagher (2007) Un nouvel enjeu pour Doha : ré-introduire le développement, L'économie politique, $\mathrm{n}^{\circ}$ 35, pp. 27 36.

GATT (1994) Résultats des négociations commerciales multilaterals du cycle d’Uruguay, Genève, GATT.

GRAHAM E., LAWRENCE R. (1996) Measuring the International Contestability of Markets. A Conceptual Approach, Journal of World Trade, vol. 30, n 5, 5-20.

Grossman G. M., Krueger A. (1993) Environmental Impacts of a North American Free Trade Agreement, in P.M. Garber (ed.) The US-Mexico Free Trade Agreement, Cambridge, MIT Press.

Hertel T. W., Winters A., eds. (2005) Putting Development Back into the Doba Agenda: Poverty Impacts of a WTO Agreement, Washington, World Bank.

Hoeckman G. (2005) The WTO and Sustainable Development, New York, United Nations University Press.

Hoeckman B., Kostecki M. (2009) The Political Economy of the World Trading System. The WTO and Beyond, $3^{\text {rd }}$ edition, Oxford, Oxford University Press.

Ismail F. (2007) Misconceptions and Myths about the WTO, Trade, Development and Aid, Note $\mathrm{n}^{\circ} 4$, Paris, IDDRI.

IsmAIL F. (2005) Mainstreaming Development in the World Trade Organization, Journal of World Trade, vol. 39, $n^{\circ} 1$, 11-21.

JONES K. (2010) The Doha Blues. Institutional Crisis and Reform in the WTO, Oxford, Oxford University Press.

KRUGMAN P. R. (1997) What Should Trade Negotiators Negotiate About?, Journal of Economic Literature, vol. XXXV, March, 113-120. 
LEKAlis J. N. (2000) Environment and Development in a Southern European Country: Which Environmental Kuznets Curves?, Journal of Environmental Planning and Managing, vol. 43, n 1, 139-153.

Mattoo A., Subramanian A. (2008) Multilateralism Beyond Doha, Working Paper $\mathrm{n}^{\circ} 153$, Washington DC, Center for Global Development.

Minor P., Tsiga M. (2008) Impact of Better Trade Facilitation in Developing Countries. Analysis with a new GTAP Database for the Value of Time in Trade, GTAP $11^{\text {th }}$ Annual Conference, Helsinki, May.

Nordström H., VAughan S. (1999) Commerce et environnement, dossiers spéciaux de l’OMC n 4, Genève, OMC.

OMC (2001) Déclaration de Doha. Programme de Doha pour le Développement, Genève, OMC.

OMC (2004) Le commerce et l'environnement, Genève, OMC.

OMC, PNuE (2009) Commerce et changement climatique, Genève, OMC-PNUE.

Ostry S. (2007) Trade, Development, and the Doha Development Agenda, in R. Wilkinson, D. Lee (eds.) The WTO after Hong Kong. Progress and Prospects for the Doha Development Agenda, London, Routledge.

Panagariya A. (2002) Developing Countries at Doha: A Political Economy Analysis, The World Economy, vol. 25, $\mathrm{n}^{\circ}$ $9,1205-1233$.

Petersmann E.U., ed. (2005) Reforming the World Trading system: Legitimacy, Efficiency and domestic Governance, Oxford, Oxford University Press.

Pisani E., Chatellier V. (2010) La faim dans le monde, le commerce et les politiques agricoles, Revue française d'économie, vol. XXV, n 1, 3-77.

POLSKI S. (2006) Winners and Losers: Impact of the Doha Round on Developing Countries, Washington DC, Carnegie Endowment for International Peace.

PorTer R. B., ed. (2001) Efficiency, Equity and Legitimacy. The Multilateral Trading System at the Millennium, Washington, Brooking Institution Press.

STEGER D., ed. (2010) WTO. Redesigning the World Trade Organization for the Twenty-first Century, Ottawa, Wilfrid Laurier University Press.

TAYLOR I. (2007) The Periphery Strickes Back? The G20 at the WTO, in R. Wilkinson, D. Lee (eds.) The WTO after Hong Kong. Progress and Prospects for the Doha Development Agenda, London, Routledge.

TisDell C. (2000) Globalisation, WTO and Sustainable Development, Economics, Ecology and the Environment Working Papers $n^{\circ} 46$, Brisbane, The University of Queensland.

WARWICK COMmission (2007) The Multilateral Trade Regime: Which Way forward, Coventry, University of Warwick.

Winham G. R. (2007) The Doha Round and its Impact on the WTO , in R. Wilkinson, D. Lee (eds.) The WTO after Hong Kong. Progress and Prospects for the Doha Development Agenda, London, Routledge.

Wilkinson R. (2007) The WTO After Hong Kong. Setting the Scene for Understanding the Round, in R. Wilkinson, D. Lee (eds.) The WTO after Hong Kong. Progress and Prospects for the Doha Development Agenda, London, Routledge.

WTO (2004) The Future of the WTO: Addressing Institutional Challenges in the New Millennium, Geneva, WTO.

Yusuf S. et al. (2007) China and India Reshape Global Industrial Geography, in L. A. Winters, S. Yusuf, Dancing with Giants: China, India and the global Economy, Washington, World Bank Institute of Policy Studies. 